Revue

de l'histoire des religions
Revue de l'histoire des religions

$4 \mid 2014$

L'objet rituel. Concepts et méthodes croisés

\title{
Les marqueurs de pain, objets rituels dans le christianisme antique et byzantin
}

Breadstamps, Ritual Objects in Early and Byzantine Christianity

\section{Béatrice Caseau}

\section{CpenEdition}

\section{Journals}

Édition électronique

URL : http://journals.openedition.org/rhr/8318

DOI : $10.4000 /$ rhr.8318

ISSN : 2105-2573

Éditeur

Armand Colin

Édition imprimée

Date de publication : 1 décembre 2014

Pagination : 599-617

ISSN : 0035-1423

\section{Référence électronique}

Béatrice Caseau, "Les marqueurs de pain, objets rituels dans le christianisme antique et byzantin »,

Revue de l'histoire des religions [En ligne], 4 | 2014, mis en ligne le 01 décembre 2017, consulté le 20 avril 2019. URL : http://journals.openedition.org/rhr/8318 ; DOI : 10.4000/rhr.8318 


\section{Les marqueurs de pain, objets rituels dans le christianisme antique et byzantin}

La catégorie des objets rituels n'inclut pas tout objet à motif religieux, mais les objets activement impliqués dans un ou des rituels religieux qu'ils soient personnels, familiaux ou communautaires, qu'ils relèvent du monde des sanctuaires ou de la sphère domestique. Cet article se penche plus particulièrement sur les marqueurs de pain qui servaient à marquer les pains d'eulogie. Ils ne font pas partie des objets du culte et relèvent plutôt du monde des objets domestiques, qui sont régulièrement impliqués dans un rituel donnant au pain une valeur religieuse. La catégorie de l'objet rituel permet de sortir du binôme objet sacré des sanctuaires / objet domestique à motif religieux pour permettre de trouver leur place à une catégorie d'objets qui fait le lien entre les deux mondes du sanctuaire et de la maison.

\section{Breadstamps, Ritual Objects in Early and Byzantine Christianity}

The category of ritual objects is large but does not include all objects bearing religious iconography, unless they are actively involved in a ritual. Can be defined as ritual objects those kept in shrines or homes and used in religious rituals, whether they are carried out by a person, a family or a community. This article focuses specifically on breadstamps used to mark the loaves offered in eulogy to churches by the faithful or to the faithful by the clergy of sanctuaries in the late antique and Byzantine periods. They are not cultic objects but rather household items that give the bread a religious value. The category of ritual object is useful for making sense of a class of objects linking the two worlds of the sanctuary and the home, opening a space between sacred objects and domestic items bearing religious iconography. 
Objets rituels, objets cultuels, objets sacrés sont trois catégories différentes, qui se recoupent en partie. Les travaux de I. Patera ont attiré l'attention sur le fait que dans les sanctuaires grecs antiques, tous les objets n'étaient pas des objets sacrés et qu'à côté de ceux directement utilisés dans le culte, il fallait aussi porter attention à des objets comme les lampes ou les offrandes ${ }^{1}$. Le recours à la catégorie d'objet rituel, par opposition à celui de vaisselle sacrée, permet en effet de rendre compte de manière plus fine de la culture matérielle religieuse en incluant à côté des objets de culte, qui reçoivent un culte ou qui servent au culte, des objets du sanctuaire jouant un rôle passif et cependant indispensable, comme les offrandes consommables, qui sont des objets rituels non pérennes mais avec un statut particulier et une consommation ritualisée, ou encore certaines offrandes permanentes, comme les ex-voto. La catégorie des objets rituels inclut aussi des objets domestiques qui n'ont pas reçu de sacralisation, n'appartiennent pas aux sanctuaires et qui cependant sont utilisés dans le cadre de rituels religieux. On tend à placer dans cette catégorie tous les objets qui semblent relever des pratiques magiques, mais la catégorie de l'objet rituel dépasse largement la magie ${ }^{2}$. Dans un article paru en 1996, la préhistorienne Ruth Whitehouse, rappelle la blague qui consiste lors de fouilles à qualifier d'objet rituel tout objet dont on ne comprend pas la fonction. Elle souligne que le terme même d'objet rituel est souvent utilisé de manière vague, sans définition, ni réflexion et propose

1. Ioanna Patera, Offrir en Grèce ancienne. Gestes et contextes, Stuttgart, Steiner Verlag (PAwB, 41), 2012; Id., «Light and Lighting Equipment in the Eleusinian Mysteries. Symbolism and Ritual Use», Light and Darkness in Ancient Myth and Religion, éd. Menelaos Christopoulos, Efimia D. Karakantza, Olga Levaniouk, Lanham, Lexington Books, 2010, p. 261- 275; Id., «Ritual Practice and Material Support: Objects in Ritual Theories», CHS Research Bulletin, 1, 2012.

http://nrs.harvard.edu/urn3:hlnc.essay:PateraI.Ritual_Practice_and_Material_ Support.2012

2. On laissera de côté dans cet article les amulettes et autres objets utilisés dans le cadre de pratiques magiques. Je renvoie simplement pour la bibliographie à l'ouvrage édité par Luke Lavan, Ellen Swift, Toon Putzeys, Objects in Context, Objects in use. Material Spatiality in Late Antiquity, Leyde, Brill («Late Antique Archaeology », 5), 2007. 
une typologie des objets rituels, construite à partir de l'archéologie préhistorique et classique qui distingue six types d'objets rituels ${ }^{3}$ :

$1 /$ les sacra, c'est-à-dire les objets qui reçoivent un culte

2/ les objets votifs, qui représentent, d'une manière ou d'une autre, le dévot donateur et qui sont des objets offerts aux divinités

3 / les offrandes, une large catégorie d'objets offerts à la divinité pour son usage ou sa glorification

4/ les objets utilisés dans les rites

5/ les dépôts funéraires, c'est-à-dire les objets déposés avec et auprès des morts

$6 /$ les amulettes.

Cette typologie peut-elle s'appliquer aussi au christianisme ancien et médiéval ? Les deux dernières catégories, celle des dépôts funéraires et des amulettes, peuvent être reprises sans modification, car il y a une continuité dans l'usage de ce genre d'objets pour accompagner les morts dans l'au-delà et pour protéger les individus, même si des différences très importantes existent entre des tombes quasiment dépourvues d'objets et des tombes richement dotées. La pertinence des quatre autres catégories est moins évidente en contexte chrétien. Ainsi, les reliques des saints font partie à la fois des objets funéraires, de ceux qui sont utilisés dans des rites comme les processions et de ceux qui reçoivent une forme de sacralisation et même un culte. Pour les sacra, on peut considérer que le pain du rituel eucharistique, pain consacré sur l'autel et devenu le corps du Christ, reçoit un culte, mais c'est aussi une offrande et un objet utilisé dans le rite. Une icône peut recevoir des gestes de vénération et entrer dans la catégorie des objets qui reçoivent un culte, en particulier dans la sphère byzantine, mais c'est aussi un objet utilisé dans le cadre de rituels liturgiques ou domestiques, ce peut être une offrande et même un objet votif quand le ou les donateurs se font représenter dessus. La vaisselle liturgique offerte par des fidèles qui font inscrire leur nom relève à la fois de l'offrande et de l'objet votif, et elle est entourée de règles qui la font inclure dans les objets sacrés. On constate donc des recoupements qui font que la typologie proposée par Ruth Whitehouse ne fonctionne pas

3. Ruth Whitehouse, «Ritual Objects. Archaeological Joke or Neglected Evidence?», Approaches to the Study of Ritual. Italy and Ancient Mediterranean, éd. John B. Wilkins, Londres ( « Accordia Specialist Studies on the Mediterranean »), 1996, p. 9-30. 
parfaitement en contexte chrétien, même si chacune des catégories existe effectivement.

On peut définir l'objet rituel comme servant dans un ou des rituels religieux, qu'ils soient personnels, familiaux ou communautaires, qu'ils relèvent du monde des sanctuaires ou de la sphère domestique. Toutefois cette définition très inclusive mérite d'être précisée. Il importe dans un premier temps de distinguer objet rituel et objet appartenant à un sanctuaire, objet rituel et geste rituel, et finalement de replacer l'objet rituel par rapport aux objets comportant un décor religieux.

\section{OBJETS RITUELS? LES ZONES GRISES}

\section{Les objets appartenant aux sanctuaires}

La catégorie des objets rituels est vaste mais n'inclut pas tout objet à motif religieux, même s'il se trouve dans un sanctuaire. Dans le monde chrétien de la fin de l'Antiquité et du Moyen Âge, le fait qu'un objet appartienne à un sanctuaire augmente ses chances d'être un objet rituel, mais la propriété ecclésiastique n'est pas un critère suffisant. Un vitrail ou un décor de chapiteau n'est pas un objet rituel, à moins d'être l'objet de prières particulières ou de gestes spécifiques. Les portes des cathédrales, par exemple, ne sont pas des objets rituels, même si elles sont impliquées dans des rites d'ouverture et de fermeture qui sont des éléments du rituel. Toutefois, elles peuvent devenir objet rituel quand une zone particulière est touchée par les fidèles, dans le cadre d'un geste de prière ou de protection personnelle. C'est le cas de certaines sculptures à la porte des églises dont la patine indique qu'elles sont touchées par les fidèles qui se rendent dans l'édifice. On en trouve un exemple à la cathédrale de Milan dont on peut considérer que la porte est alors devenue un objet rituel, à cause du geste répété des fidèles.

Dans le christianisme, la croix est par excellence une image symbolique, qui joue un rôle central pour donner une identité chrétienne à un lieu, qu'il s'agisse d'une maison, d'une tombe, d'une église. Si toutes les croix peintes ou en mosaïque ne sont pas des objets rituels, mais forment le décor d'un sanctuaire, on 
peut cependant admettre dans cette catégorie les croix insérées dans les colonnes de certaines églises comme Saint-Jean d'Ephèse, ou Sainte-Sophie de Constantinople ${ }^{4}$ (fig. 1). Certaines de ces croix encastrées dans les colonnes contenaient en effet des reliques, comme par exemple au monastère Sainte-Catherine du Sinaï et des prières étaient prononcées devant elles dans le cadre du culte des saints 5 .

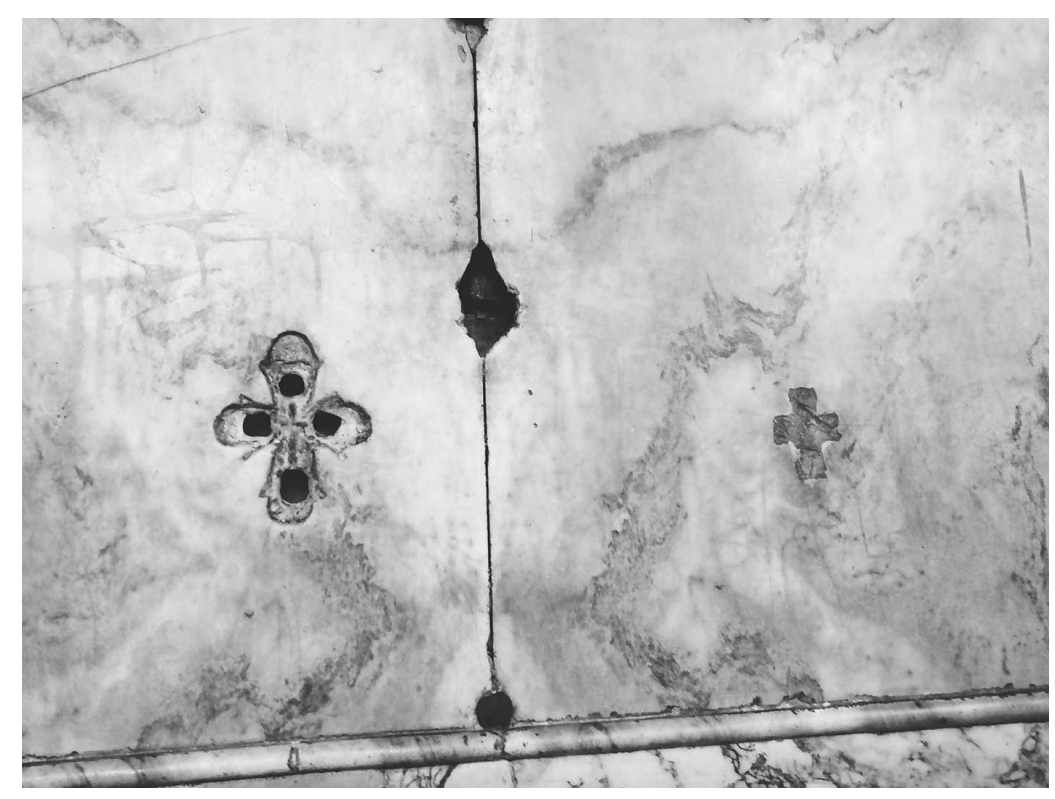

Fig. 1 : Traces de croix votives insérées dans le marbre dans une galerie de la basilique Sainte-Sophie de Constantinople (cliché B. Caseau).

Le christianisme ancien est un bon terrain d'observation pour réfléchir sur l'objet rituel, dans la mesure où l'on constate une progressive différentiation entre objets domestiques et vaisselle liturgique, réservée à un usage rituel. Au fur et à mesure que le culte chrétien se met en place, les objets impliqués dans les rituels chrétiens, en particulier lors du repas eucharistique, se diversifient

4. Natalia Teteriatnikov, «Devotional Crosses of Hagia Sophia», Byzantion, 68, 1998, p. 419-445.

5. George H. Forsyth, Kurt Weitzmann, The Monastery of St. Catherine at Mount Sinai. The Church and Fortress of Justinian, Ann Arbor, University of Michigan Press, 1973, pl. XLIII. 
et connaissent un processus de mise à part qui correspond aux premières étapes de leur sacralisation. Ils cessent d'avoir plusieurs usages pour ne plus servir que sur l'autel qui cesse d'être une table ordinaire pour être une table consacrée par l'évêque ${ }^{6}$. La vaisselle liturgique doit aussi être séparée de la vaisselle ordinaire. Elle est composée d'objets spécialisés pour un usage particulier à un moment du rituel. Le choix de matériaux nobles, en particulier l'argent, est visible dans les trésors d'objets liturgiques qui ont été retrouvés enfouis 7 . Un épisode de la Vie de Théodore de Sykéôn au $\mathrm{VII}^{\mathrm{e}}$ siècle montre qu'on a le souci de trouver des objets en argent mais qu'il faut veiller à la pureté de l'usage des objets ${ }^{8}$. Il semble qu'à cette date, on puisse d'emblée utiliser les objets achetés, sans plus ample bénédiction. Cependant, il existe un gradient de sacralité dans ces objets liturgiques. Certains, soit qu'ils aient été soumis à un rituel de sacralisation par l'évêque, soit que leur utilisation sur l'autel et leur contact avec le corps et le sang du Christ les ait sacralisés, ont un statut particulier. Leur accès est réservé à un nombre limité de clercs : l'évêque, le prêtre ou le diacre. Le concile de Laodicée interdit aux sous-diacres de bénir le calice ou de donner des pains d'eulogie'. Toute la vaisselle liturgique n'est toutefois pas impliquée dans cette haute sacralisation et il existe aussi des objets de la vaisselle liturgique que des clercs mineurs peuvent toucher, comme les encensoirs ou les lampes par exemple.

$\mathrm{Si}$, grâce aux inventaires des biens des églises, on peut se faire une idée de la diversité de ces objets que possédaient les églises chrétiennes, doit-on pour autant considérer comme objet rituel tout

6. Rituel de dédicace décrit dans l'euchologe Barberini datant du viII ${ }^{\mathrm{e}}$ siècle: Stefano Parenti et Elena Velkovska éd., L'Eucologio Barberini gr. 336, Rome, Centro Liturgico Vincenziano, 2000, $\$ 151$ p. 159-162. Sur l'usage de consacrer une église et son autel avec des reliques, Vasileios Marinis, Architecture and Ritual in the Churches of Constantinople. Ninth to Fifteenth Centuries, Cambridge, Cambridge University Press, 2014, p. 28-30.

7. Susan A. Boyd, Marlia Mundell Mango éd., Ecclesiastical Silver Plate in Sixth-Century Byzantium, Washington, Dumbarton Oaks, 1993; M. Mundell Mango, «The Origins of the Syrian Ecclesiastical Silver Treasures of the SixthSeventh Centuries », Argenterie romaine et byzantine. Actes de la Table ronde Paris 11-13 octobre 1983, éd. François Baratte, Paris, 1988, p. 163-178.

8. Vie de Théodore de Sykéôn, 42, trad. éd. André-Jean Festugière, Bruxelles, 1970, p. 39-40.

9. Concile de Laodicée, c. 25, Les canons des synodes particuliers, t. I, 2. Discipline générale antique (IVe-IX $s$.$) , Pierre-Périclès Joannou, Grotteferrata,$ 1962, p. 141. 
le contenu de ces inventaires ${ }^{10}$ ? Doit-on par exemple distinguer dans les objets liturgiques ceux qui sont soumis aux gestes rituels de manière répétée et ceux qui sont conservés dans un trésor sans être utilisés?

\section{Objet rituel et geste rituel}

Pour entrer dans la catégorie des objets rituels, l'objet doit donc être activement impliqué dans le rituel religieux ${ }^{11}$. Les croix qui servent en tête des processions, à l'intérieur ou à l'extérieur des églises ou qui reçoivent la vénération des fidèles, font naturellement partie des objets rituels, ainsi que les enkolpia, objets souvent en forme de croix portés autour du cou ou sur la poitrine, tant par les ecclésiastiques que par les laïcs, dont certains contenaient des reliques de saints et étaient rituellement embrassés. Mais que signifie être activement impliqué dans le rituel? Il faut sans doute inclure dans la définition un usage récurrent. Dans la cité d'Apamée, des croix ont été gravées sur les colonnes effondrées lors des tremblements de terre de 526 et 528 , pour que la colonnade résiste, une fois redressée, au prochain séisme ${ }^{12}$. Il y a eu sur ces colonnes un geste rituel de protection des lieux, mais cela ne fait pas des colonnes des objets rituels. Nous n'avons pas d'attestation que les croix de ces colonnes aient été vénérées par les habitants. Elles étaient trop haut placées sur les colonnes pour servir à un rituel. De surcroît, des croix se trouvaient partout sur les bâtiments de la ville, en trop grand nombre pour que chacune ait reçu de l'attention. Ces croix d'Apamée, tout comme de nombreuses autres ornant l'encadrement des portes de maisons, avaient une fonction apotropaïque. Graver la croix était un geste rituel, mais ce geste ne

10. Béatrice Caseau, «Objects in Churches: the Testimony of Inventories», Objects in Context, Objects in Use, p. 551-579; Brigitte Pitarakis, «La vaisselle eucharistique dans les églises d'Orient», Pratiques de l'eucharistie dans les Églises d'Orient et d'Occident (Antiquité et Moyen Âge), éd. Nicole Bériou, Béatrice Caseau, Dominique Rigaux, Paris, Institut d'Études Augustiniennes («Collection des études augustiniennes», 45), 2009, p. 309-329.

11. Catherine M. Bell, Ritual Theory, Ritual Practice, New York, Oxford University Press, 1992, p. 38-46; Stanley Tambiah, «A Performative Approach to Ritual», Proceedings of the British Academy, 65, 1979, p. 113-169.

12. Jean-Charles Balty, «Apamée au $\mathrm{VI}^{\mathrm{e}}$ siècle. Témoignages archéologiques de la richesse d'une ville», Hommes et richesses dans l'Empire byzantin, t. 1, Paris, 1989, p. 79-96. 
transformait pas pour autant l'objet marqué en objet rituel. En effet, la dédicace d'une maison ou d'un lieu par la prière et la gravure de croix ne fait pas de la maison ou de l'église un objet rituel. Si l'offrande peut transformer un objet domestique en objet rituel, on ne peut étendre cela aux bâtiments, même si la gravure, peinture, sculpture de croix a une fonction religieuse de protection et qu'elle est ensuite accompagnée par un rituel de dédicace avec des prières accompagnées de gestes rituels. Dédicace et offrande se ressemblent dans l'esprit, mais la zone du bâtiment où se trouve la gravure de croix ne peut-être considérée comme objet rituel que si d'autres cérémonies se développent autour de ladite gravure, sculpture ou peinture.

Parmi les gestes rituels qui modifient le statut de l'objet, il faut faire une place aux offrandes. Il faut sans doute restreindre aux objets mobiles la catégorie de l'objet devenu rituel par une offrande à Dieu et à ses saints ${ }^{13}$. Ainsi, les lampes mobiles apportées lors de processions et parfois déposées dans les sanctuaires, les cierges allumés par les fidèles devant un reliquaire, une icône ou une statue devant lesquelles la prière s'organise, relèvent de l'objet rituel, mais on hésite pour les lampes fixes et les lustres des sanctuaires, car ce sont des témoins passifs. Les typika monastiques incluent des recommandations sur l'éclairage en temps de fête, ce qui montre son importance ${ }^{14}$. Les ekphraseis, comme le poème de Paul le Silentiaire sur Sainte-Sophie, ne manquent pas de souligner le nombre des lampes et leur capacité à illuminer le sanctuaire ${ }^{15}$. Il existe de surcroît un personnel qui s'occupe de remplir d'huile les polykandela pour que l'espace ecclésial soit éclairé pour les cérémonies religieuses et plus encore pour les jours de fête. Il existe donc une zone grise pour ces objets fixes, car si le remplissage, nettoyage et allumage de ces lampes ne se produit pas pendant le rite religieux lui-même, il se fait en préparation à ce dernier pour

13. Statutairement, les biens immobiliers donnés à Dieu et à ses saints sont soustraits de la circulation des biens et ont un statut juridique particulier.

14. Eleanor A. Congdon, «Imperial Commemoration and Ritual in the Typikon of the Monastery of Christ Pantokrator», Revue des études Byzantines, 54, 1996, p. 161-199.

15. Paul le Silentiaire, Description de Sainte-Sophie de Constantinople, 806889, trad. M.-Ch. Fayant et P. Chuvin, Die, 1997, p. 112-118; Laskarina Bouras, Maria G. Parani, Lighting in Early Byzantium, Washington, Dumbarton Oaks, 2008, p. 31-36. 
embellir la fête. On peut donc considérer que ces objets jouent un rôle important dans le bon déroulement du rituel et ils donnent à ce dernier l'une de ses caractéristiques symboliquement importante: la lumière ${ }^{16}$. Toutefois, si l'on peut concevoir d'admettre les lampes fixes, les lustres et les polykandela dans les objets rituels, cela ne doit pas conduire à faire entrer dans cette catégorie tout objet présent dans le sanctuaire au moment du rituel, ou tout objet appartenant au sanctuaire. La propriété ecclésiastique n'est pas un critère suffisant pour établir qu'un objet relève des objets rituels, il faut qu'il ait une fonction dans l'un au moins des rituels.

\section{Les objets à motifs religieux}

À la fin de l'Antiquité, le nombre des objets portant un motif religieux chrétien s'est étoffé au point d'englober non seulement les objets liturgiques mais aussi des objets de la vie courante. Tout objet qui porte une iconographie religieuse n'est pas un objet rituel, il faut encore qu'il joue un rôle dans les rituels liturgiques et paraliturgiques. Mais où se fait la frontière? Entre le $\mathrm{IV}^{\mathrm{e}}$ et le $\mathrm{VII}^{\mathrm{e}}$ siècle, on constate une christianisation des objets domestiques qui se manifeste par une ornementation avec des symboles comme la croix ${ }^{17}$ ou d'autres motifs chrétiens. On constate parallèlement, mais avec une relative lenteur, l'élimination progressive de scènes liées à la mythologie ou aux cultes traditionnels du monde grécoromain. Ainsi la présence de croix, ou d'un motif iconographique inspiré de la Bible, sur la vaisselle de table à la fin de l'Antiquité comporte une allusion à l'identité religieuse de son propriétaire mais ne fait pas pour autant de cette vaisselle un objet rituel.

La simple gravure de croix donne à tout objet d'être l'objet d'un rituel un bref moment, parce que le geste lui-même est porteur d'un message religieux et peut avoir été réalisé dans ce cadre mental qui associe la croix à l'exorcisme, à la protection et à l'histoire du Salut. Mais toute lampe portant une croix ou une image religieuse

16. Claire Nesbitt, «Shaping the Sacred: Light and the Experience of Worship in Middle Byzantine Churches », Byzantine and Modern Greek Studies, 36, 2012, p. $139-160$.

17. Sur les différents supports qui portent des croix: Erich et Erika Dinkler, «Kreuz I», Reallexikon zur byzantinischen Kunst, 5, 1995, p. 1-219 (avant l'iconoclasme); George Galavaris, «Kreuz II», ibidem, p. 219-284 (après l'iconoclasme). 
n'est pas un objet rituel, affirmer le contraire reviendrait à fusionner la catégorie d'objet rituel avec celle d'objets à motifs religieux et à donner une fonction rituelle à ce qui peut être simplement ornemental. En fait, on touche là à une autre zone grise liée à la polysémie des objets à motifs religieux. Pour transformer la lampe à motif de croix en objet rituel, il faut sans doute plus que la prière de l'artisan qui grave la croix, et plus que la prière automatique qui peut surgir à la vue d'une croix dans les milieux chrétiens les plus pieux; il faut que la lampe soit associée, de manière régulière ou ponctuelle, à une cérémonie et donc au monde des sanctuaires ou, dans la maison, à la zone du culte domestique. Ainsi les lampes à huile qui portent des inscriptions religieuses comme «lumière du Christ qui éclaire tout» pouvaient être achetées dans le cadre d'un pèlerinage et rapportées chez soi pour bénéficier de la sacralité du lieu visité ${ }^{18}$.

Pour l'archéologue, il est très difficile, voire impossible de distinguer parmi ces objets domestiques à motifs religieux et ceux qui sont des objets rituels. La vaisselle de table ordinaire, les amphores transportant ou stockant des denrées alimentaires, pouvaient être ornées de croix, par exemple, dans un souci de protéger globalement par ce signe apotropaïque l'alimentation de la maison et au-delà le propriétaire de l'objet ${ }^{19}$. Ainsi, la présence d'une croix sur une amphore n'en fait pas un objet rituel. Toutefois, parmi ces objets domestiques, certains provenaient de sanctuaires, qu'il s'agisse de souvenirs de pèlerinage ou d'objets permettant de participer aux rituels liturgiques; d'autres étaient achetés ou créés pour en faire des offrandes. Ces deux dernières catégories d'objets relèvent des objets rituels. Quelques quatre mille lampes de terre cuite ont ainsi été trouvées dans la fontaine aux lampes à Corinthe: ces lampes, dont certaines portent un motif chrétien, étaient offertes dans le cadre de rituels souvent qualifiés de magiques par les commentateurs, mais qui relèvent plus simplement - et sans

18. Stanislao Loffreda, Lucerne bizantine in Terra Santa con iscrizioni in Greco, Jerusalem, Franciscan Printing Press («Studium Biblicum Franciscanum», 35), 1989, p. 79-120.

19. Eunice Dauterman Maguire, Henry P. Maguire, Maggie J. DuncanFlowers, Art and Holy Powers in the Early Christian House, Urbana-Champaign, University of Illinois Press, 1989, p. 20-21 et n. 63 p. 132; fonction apotropaïque des croix: Christopher Walter, «IC XC NI KA: The Apotropaic Function of the Victorious Cross », Revue des études Byzantines, 55, 1997, p. 193-220. 
jugement de valeur - de rituels religieux ${ }^{20}$. Qu'il s'agisse de la manifestation d'attachement aux anciens cultes désormais interdits aux $\mathrm{v}^{\mathrm{e}}$ et $\mathrm{VI}^{\mathrm{e}}$ siècles, ou de rituels de protection en des lieux jugés propices à la communication avec des êtres spirituels comme les anges, ou les deux à la fois, ces offrandes de lampes ou de pièces de monnaie n'étaient pas anodines. Elles opéraient sur les objets un changement de nature puisque par l'offrande, ils passaient d'objet domestique à objet rituel.

Le lien avec le sanctuaire se révèle donc important pour identifier des objets courants comme objets rituels. On peut se poser, par exemple, la question pour certaines cuillers inscrites portant un décor de croix et des références à un vœu ou à la prière ${ }^{21}$, ou encore pour ces cuillers faisant référence à saint Jean et à la manne ${ }^{22}$. Même si des cuillers pour mélanger le vin et l'eau se trouvent dans les trésors d'objets liturgiques, ces cuillers particulières n'étaient sans doute pas des objets de culte ${ }^{23}$. Ces cuillers domestiques semblent provenir d'un sanctuaire, et appartenir à la catégorie des objets de pèlerinage $e^{24}$, donc relever des objets rituels.

\section{Entre la maison et le sanctuaire : les marqueurs de pain}

Parmi ces objets domestiques que l'on peut placer dans la catégorie des objets rituels, on peut faire une place particulière aux marqueurs de pain. Michael Grünbart estime à environ cinq cents les marqueurs, en bois, en terre cuite ou en métal dans les différents

20. Karen S. Garnett, «Late Roman Corinthian Lamps from the Fountain of the Lamps», Hesperia, 44, 1975, p. 173-206.

21. Marlia Mundell Mango, Silver from Early Byzantium. The Kaper Koraon and Related Treasures, Baltimore, The Walters Art Gallery, 1986, p. 118-127.

22. Arietta Papaconstantinou, «La manne de saint Jean. À propos d'un ensemble de cuillers inscrites», Revue des études Byzantines, 59, 2001, p. 239246.

23. Robert Taft, «Byzantine Communion Spoons: a Review of the Evidence», Dumbarton Oaks Papers, 50, 1996, p. 209-238; B. Caseau, "L'abandon de la communion dans la main ( $\mathrm{IV}^{\mathrm{e}}-\mathrm{XII}^{\mathrm{e}}$ siècles)», Travaux et mémoires (Mélanges Gilbert Dagron), 14, 2002, p. 79-94 ; François Baratte, «La vaisselle d'argent dans l'Afrique romaine et byzantine », Antiquité Tardive, 5, 1997, p. 111-132 (Augustin possédait des cuillers en argent d'après son hagiographe, Possidius).

24. Gary Vikan, Early Byzantine Pilgrimage Art, Revised Edition, Washington, 2010; Id., "Early Byzantine Devotionalia as Evidence of Appearance of Pilgrimage Shrines », Sacred Images and Sacred Power in Byzantium, Aldershot, Ashgate, 2003, VI. 
musées et chez des collectionneurs ${ }^{25}$. Ces objets portent des noms divers dans la littérature moderne, mais ils sont souvent appelés breadstamps ou brot stempel parce qu'on supposait que leur fonction première était de marquer la pâte à pain avant sa cuisson dans le four. En fait, les marqueurs ne servaient pas seulement à marquer le pain mais aussi d'autres supports avant cuisson, comme les amphores, les lampes en terre cuite ou les briques. En français, on utilise parfois le mot de sceau pour les désigner, car ils impriment une marque sur le support qu'ils touchent en y laissant leur empreinte ${ }^{26}$. Le geste de marquer un objet avant sa cuisson pour lui donner non seulement un décor mais aussi une identité est un geste rituel, qui est opéré de manière régulière et répétitive à la maison ou dans l'atelier artisanal et ensuite dans les cuisines des monastères et des sanctuaires.

Dans le monde romain, l'usage de tels marqueurs était très répandu et entrait dans le cadre des conduites sociales dont parlait John Scheid quand il définissait indirectement les rites comme conduites sociales, gestes et obligations ${ }^{27}$. Les marqueurs de l'époque romaine avaient des fonctions diverses puisqu'ils étaient utilisés aussi bien dans le cadre du commerce que dans le cadre cultuel. Ils ont été créés pour permettre des gestes que l'on peut qualifier de rituels, mais qui ne sont pas nécessairement religieux. Quand il ne se réduit pas à un nom, celui du propriétaire de l'objet ou celui de son fabricant, leur message est polysémique et fonctionne en effet à plusieurs niveaux, allant du pratique au symbolique, du factuel au magico-religieux ${ }^{28}$. L'absence de références religieuses dans un grand nombre d'entre eux durant le Haut-Empire cède la place à la présence systématique

25. Michael Grünbart, «Byzantine Metal Stamps in a North American Private Collection », Dumbarton Oaks Papers, 60, 2006, p. 13-24.

26. B. Caseau, «Sceaux», Antiquités paléochrétiennes et byzantines des collections du musée d'art et d'histoire, éd. Marielle Martiniani-Reber, Genève («Collections byzantines du MAH de Genève», 2), 2011, p. 178-183.

27. John Scheid, «Le sens des rites. L'exemple romain», Rites et croyances dans les religions du monde romain, Vandoeuvres - Genève, Fondation Hardt, 2007, p. 39-64; id., Quand faire, c'est croire. Les rites sacrificiels des Romains, Paris, 2005.

28. B. Caseau, «Magical Protection and Stamps in Byzantium», Seals and Sealing Practices in the Near East. Developments in Administration and Magic from Prehistory to the Islamic Period, Proceedings of the International Workshop in Cairo, 2-3 December 2009, éd. Ilona Regulski, Kim Duistermaat, Peter Verkinderen, Louvain («Orientalia Lovaniensia Analecta», 219), 2012, p. 119137. 
de croix dans ceux qui sont créés à la fin de l'Antiquité en contexte chrétien. Les marqueurs permettent donc de mesurer la recherche d'une protection religieuse pour les gens comme pour les objets, protection qui s'opère à cette date par la gravure de la croix ou par l'inscription d'une prière.

Les marqueurs ont continué à être utilisés sur des objets en terre cuite, mais certains servaient à marquer le pain avant sa cuisson. L'usage est très ancien et permettait d'identifier et de reprendre son pain dans des fours partagés. Au-delà de cet usage pratique, en contexte chrétien, les marqueurs ont aussi un but religieux, permettant la bénédiction du pain, comme l'a bien montré George Galavaris $^{29}$. On a eu tendance dans le passé à voir dans tout marqueur portant une croix un sceau eucharistique mais c'est ne pas comprendre l'usage très étendu des marqueurs dans la sphère domestique comme dans celle des sanctuaires ${ }^{30}$. Les différentes catégories de marqueurs comportant un motif religieux chrétien et parfois une inscription, attiraient sur le donateur ou le consommateur du pain la bénédiction divine et faisaient donc du pain un aliment ayant une dimension religieuse, qui n'était pas réservée au seul pain consacré sur l'autel. Elle existait pour différents types de pain allant du pain quotidien pour lequel le chrétien prie chaque jour aux pains offerts aux sanctuaires ou reçus des mains du clergé. C'est au cours de la fin de l'Antiquité que se mettent en place des usages sur ce qu'il convient de faire pour préparer le pain destiné à l'autel et pour les eulogies, les pains offerts par des fidèles à l'église et destinés à être redistribués aux membres du clergé, aux pauvres et éventuellement comme pain bénit aux fidèles eux-mêmes. Il était important pour les fidèles de faire enregistrer leur don et de bénéficier ainsi des prières eucharistiques, même si ce n'était pas leur pain qui était consacré sur l'autel. Ainsi, on peut penser que les marqueurs portant une croix et le nom du propriétaire ont pu être

29. George Galavaris, Bread and the Liturgy. The Symbolism of Early Christian and Byzantine Bread Stamps, Madison, University of Wisconsin Press, 1970, p. 110-111.

30. Vera Zalesskaya, Pilgrim Treasures from the Hermitage: ByzantiumJerusalem, éd. Lund Humphries, Amsterdam, 2005, p. 76, considère que la présence du motif de la croix est un critère suffisant: «In the middle of the medallion or the seal there is usually a cross, indicating that this is a liturgical bread»; Yannis D. Varalis, «Un sceau paléochrétien de pain eucharistique de l'agora d'Argos», Bulletin de Correspondance Hellénique, 118, 2, 1994, p. 331-342. 
utilisés pour permettre l'enregistrement du pain offert à l'église, même s'ils servaient aussi pour la cuisson du pain de table dans des fours collectifs ${ }^{31}$. Les pains d'eulogie étaient offerts dans l'attente d'une bénédiction divine comme on le voit par les inscriptions de certains marqueurs. Un marqueur conservé au British Museum à Londres porte la formule de prière «Eulogie du Seigneur sur nous et sur nos enfants » pour appeler la bénédiction sur une famille. On peut imaginer qu'il était utilisé chaque semaine pour le pain de la prosphora (fig. 2).

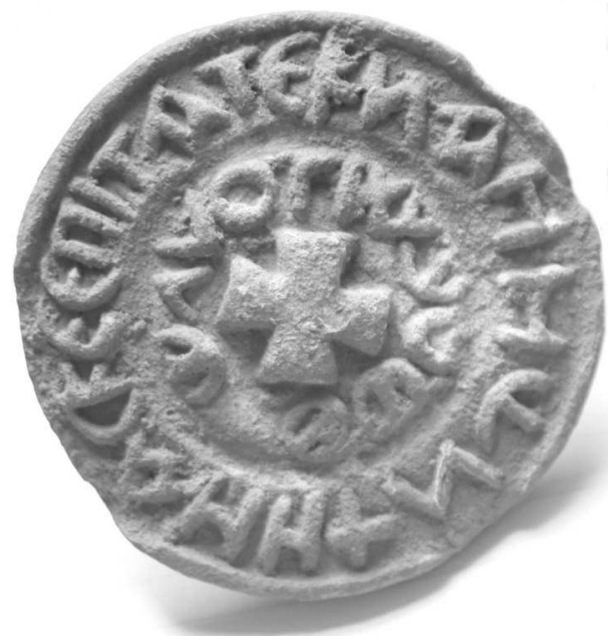

Fig. 2: Marqueur de pain, British Museum n. inv. 1995 11-7 10 (cliché B. Caseau).

Selon F. Manns, la plupart des sanctuaires fabriquaient leurs eulogies et donc possédaient leurs propres marqueurs ${ }^{32}$. Ces marqueurs portaient parfois le motif de l'église ou bien le nom du saint ou encore un symbole caractéristique. Ils étaient utilisés pour distribuer du pain, en particulier à l'occasion de la fête du saint. Les monastères assez richement dotés pouvaient accueillir à leur

31. B. Caseau, «Autour de l'autel: le contrôle des donateurs et des donations alimentaires », Donations et Donateurs dans le monde byzantin, Actes du colloque international de l'Université de Fribourg (13-15 mars 2008), éd. Jean-Marie Spieser et Élisabeth Yota, Paris, 2012, p. 47-73.

32. Frédéric Manns, «Un sceau de la transfiguration», Liber Annuus, XXV, 1975, p. 166. 
porte de nombreux visiteurs. La Vie de saint Euthyme par Cyrille de Scythopolis mentionne l'arrivée, sans doute exceptionnelle, de quatre cents Arméniens qui furent nourris avec du pain, de l'huile et du $\operatorname{vin}^{33}$. Les sources hagiographiques attestent ce rôle nourricier des monastères à l'égard des pèlerins et leur habitude de faire des distributions de pain qui étaient sans doute marqués du sceau du sanctuaire. Un marqueur en bois conservé au Cleveland Museum of Art qui représente la basilique constantinienne et l'Anastasis serait celui du sanctuaire le plus célèbre de Jérusalem ${ }^{34}$. Chaque marqueur avait un motif permettant de reconnaître sa provenance: un marqueur du Mont Thabor porte la scène de la Transfiguration ${ }^{35}$. Saint Philippe était vénéré à Hiérapolis en Phrygie où il avait un mausolée. Or un marqueur représentant Saint Philippe entouré de chaque côté par un bâtiment dont le toit est surmonté d'une croix a été ainsi précisément trouvé à Hiérapolis ${ }^{36}$.

En plus de ces marqueurs destinés aux pains d'eulogie distribués aux fidèles et aux pèlerins, les sanctuaires étaient en possession de marqueurs plus spécifiquement destinés au pain consacré sur l'autel qui devait être fractionné en petits morceaux pour la communion. On a conservé de grands marqueurs dont le décor de losanges ou de petits carrés permettaient de quadriller le pain et d'en faciliter le fractionnement pour la distribution de la communion. Les plus beaux portent aussi une inscription qui confirme leur usage eucharistique. Ces objets datent du temps où la communion des fidèles était encore assez importante pour justifier de faire cuire et de consacrer de grands pains. Il ne semble pas qu'il y ait eu un modèle unique de marqueur eucharistique pour la période de la fin de l'Antiquité. Par la suite, au Moyen Âge, dans les églises byzantines où le pain offert sur l'autel continue d'être du pain levé contrairement aux églises latines qui optent pour un pain azyme, un

33. Cyrille de Scythopolis, Vie de saint Euthyme, éd. Eduard Schwartz, Kyrillos von Skythopolis, Leipzig, 1939, p. 27.

34. Margaret E. Frazer, "Bread stamp with Holy Sepulcher», Age of Spirituality. Late Antique and Early Christian Art, Third to Seventh Century, éd. Kurt Weitzmann, The Metropolitan Museum of Art, New York, 1979, fig. 528, p. 588-589.

35. F. Manns, «Un sceau de la transfiguration», p. 146-170.

36. Anna Gonosova, Christine Kondoleon, Art of Late Rome and Byzantium in the Virginia Museum of Fine Arts, Richmond, Virginia Museum of Fine Arts, 1994, p. 270-271. 
marqueur spécifique comportant l'inscription IC XC NI KA, «Jésus Christ vainc », inscrite dans un carré ou un rectangle, s'impose pour le pain consacré sur l'autel. L'évolution du rite eucharistique, avec l'usage de la lancette, permet de découper dans un pain, avant la consécration, l'amnos, «l'Agneau de Dieu» qui sera consacré, et d'en détacher d'autres parties pour faire des commémoraisons pour la Mère de Dieu, les saints, les vivants et les morts ${ }^{37}$. Certains marqueurs reflètent cette évolution liturgique ${ }^{38}$. En Occident et en Arménie où le pain azyme est utilisé, des moules à hostie permettent de marquer l'hostie du prêtre, souvent, d'une crucifixion.

Dans le contexte des pratiques liturgiques, les marqueurs sont intéressants, car ils forment une catégorie d'objets qui ne fait pas partie des objets du culte: contrairement à la patène et au calice, ils ne viennent pas sur l'autel, pas même dans la chapelle où se fait le rite de la prothesis en Orient. Ces objets ne sont pas des objets sacrés, ils n'ont pas reçu de prières consécratoires, comme la patène et le calice. Ils ne sont pas non plus des objets cultuels puisqu'ils ne sont pas portés à l'église. Même les marqueurs spécifiques du pain à offrir sur l'autel ne font pas partie du rituel eucharistique à proprement parler mais de sa préparation. Ils sont utilisés avant la cuisson, et donc bien avant la consécration du pain. Ils ne sont donc pas en contact avec le corps du Christ. Surtout, les marqueurs ne se limitent pas aux sceaux eucharistiques. Ils servent bien plus largement dans le cadre d'une préparation ritualisée du pain, devenu un aliment pour lequel on fait des prières et pour lequel on rend grâces, même s'il n'est pas destiné à l'église. Ce ne sont pas des objets exclusivement ecclésiastiques. Les familles chrétiennes qui n'étaient pas dans la misère possédaient des marqueurs en bois, en terre cuite ou en métal, et les utilisaient sur leur pain. On peut les inclure dans le monde des objets domestiques, au même titre que la vaisselle, mais ils sont conservés parmi les objets religieux qui ont une valeur symbolique forte. Grâce aux marqueurs, même pour la

37. Robert Taft, A History of the Liturgy of St. John Chrysostom, 6 vol., Rome, 1975-2008; Marie-Hélène Congourdeau, «L'enfant immolé. Hyper-réalisme et symbolique sacrificielle à Byzance», Pratiques de l'eucharistie dans les Églises d'Orient et d'Occident, éds. Nicole Bériou, Béatrice Caseau et Dominique Rigaux, Paris: Institut des Études Augustiniennes, t. 1, Paris, 2009, p. 291-307. 2007.

38. Iglika Mishkova, Prosphora, Sophia, Musée ethnographique national, 
table quotidienne, les pains avaient acquis une dimension religieuse au-delà de leur fonction nutritive. Le message religieux imprimé par le marqueur faisait de ces pains des porteurs de bénédiction. Dans le cadre des dons de pain, on sait que chacun souhaitait que ce don ne soit pas anonyme: les familles donatrices comme les sanctuaires utilisaient donc les marqueurs pour donner au pain une identité. Les marqueurs relèvent des objets rituels qui font le lien entre la sphère domestique et celle des sanctuaires.

Il faut admettre l'utilité de la catégorie de l'objet rituel pour ce genre d'objet, car elle permet de sortir du binôme objet sacré des sanctuaires / objet domestique à motif religieux pour permettre de trouver leur place à une catégorie d'objets qui fait le lien entre les deux mondes du sanctuaire et de la maison. D'autres objets auraient pu être analysés de la même manière, comme, par exemple, les icônes qui ont été bénies sur l'autel à l'église avant d'être posées chez soi, les objets de pèlerinage qui étaient conservés à la maison mais provenaient des sanctuaires dont ils diffusaient la sacralité. Les pèlerins repartaient volontiers des sanctuaires avec des souvenirs, comme des ampoules pleines d'huile ayant brûlé sur le tombeau du saint ${ }^{39}$, ou des jetons portés sur soi et représentant le saint visité ${ }^{40}$, ou encore avec des pains marqués du sceau du sanctuaire. Il est intéressant de noter qu'on trouve sur ces différents types d'objets des formules similaires, parfois aussi un décor facilement identifiable. Ces différents objets transportés hors du sanctuaire et diffusés dans la société créaient une communauté de fidèles adeptes d'un culte de saint particulier ${ }^{41}$. On a pu mesurer le rayonnement d'un sanctuaire au nombre des ampoules retrouvées et à leur dispersion dans le monde méditerranéen. Les pains ne se sont pas conservés, mais on peut supposer une distribution assez analogue. La popularité du monastère de Saint-Ménas ne fait pas

39. Catherine Metzger, Les ampoules à eulogie du musée du Louvre, Paris, Réunion des Musées Nationaux, 1981.

40. Jetons et eulogies de saint Syméon stylite, Jean-Pierre Sodini, «Objets de dévotion de la collection Khoury », Mélanges de l'université Saint Joseph, 63, 2010-2011, p. 19-62; Richard Temple, Early Christian and Byzantine Art, Textiles, Metalwork, Frescoes, Manuscripts, Jewellery, Steatites, Stone Sculptures, Tiles, Pottery, Bronzes, Amulets, Coins and other items $4^{\text {th }}$ to the $14^{\text {th }}$ Centuries, Londres, Temple Gallery, 1990, n. 87, p. 112.

41. Sur le rapport entre objet et groupe social, Alfred Gell, Art and Agency: an Anthropological Theory, Oxford, Clarendon Press, 1998. 
de doute à la fin de l'Antiquité et on a retrouvé de nombreuses ampoules de saint Ménas dont certaines portent parfois un message de bénédiction semblable à celui des marqueurs, ce qui montre que ces objets ont en commun d'attirer et de transmettre la «bénédiction de saint Ménas $»^{42}$.

Un autre type d'objet que sont les amulettes relève aussi de la catégorie des objets rituels mais mériteraient à eux seuls un chapitre $^{43}$. La figure du cavalier terrassant une démone, tout comme celle du mauvais œil, étaient très appréciées, mais ce recours à une magie dont les racines venaient des anciens cultes païens ou du judaïsme ne pouvait se faire avec l'approbation ecclésiastique, qui proposait une autre magie, chrétienne, avec les images des saints et les ampoules d'huile sainte. Les objets transportés par les pèlerins avaient valeur d'amulettes, comme le soulignait G. Vikan ${ }^{44}$.

Il y a encore une dimension de l'objet rituel qui n'a pas été abordée: celle de son devenir. Les objets ont une histoire et parfois plusieurs identités à travers le temps. Ils peuvent changer de statut, passer d'objet séculier ou d'objet à motif religieux à objet rituel, tandis que le chemin inverse existe aussi ${ }^{45}$. Ainsi, parmi les livres liturgiques et les livres d'heures, certains sont activement utilisés lors de cérémonies religieuses ou lors de moments de recueillement,

42. Les ampoules de saint Ménas portent parfois l'inscription EULOGIA TOU AGIOU MENA, Nancy Patterson Sevcenko, «Flask with St. Thecla and orant (St. Menas ?)», in Age of Spirituality, op. cit., n. 516, p. 576-577; Monica Gilli, Le ampolle di San Mena. Religiosità, cultura materiale e sistema produttivo, Rome, Palombi, 2002; Paola Lopreato, «Le ampolle di San Menas e la diffusione del suo culto nell'Alto Adriatico», Aquileia e l'oriente mediterraneo, Udine («Antichità altoadriatiche», 12), 1977, p. 411-28; Pierre Maraval, Lieux saints et pèlerinages d'Orient. Histoire et géographie des origines à la conquête arabe, Paris, Cerf, 2004², p. 237-241; sur le sanctuaire de saint Ménas: Peter Grossmann, Abu Mina, I. Die Gruftkirche und die Gruft, Mayence, 1989; id., «The Pilgrimage Center of Abû Mînâ», Pilgrimage and Holy Space in Late Antique Egypt, éd. David Frankfurter, Leyde, Brill («Religions in the Graeco-Roman World», 134), 1998, p. 281-289.

43. John G. Gager éd., Curse Tablets and Binding Spells from the Ancient World, New York, Oxford University Press, 1992; John Mitchell, «Keeping the Demons out of the House: the Archaeology of Apotropaic Strategy and Practice in Late Antique Butrint and Antigoneia», Objects in Context, Objects in use, p. 273310.

44. Gary Vikan, «"Guided by Land and Sea". Pilgrim Art and Pilgrim Travel in Early Byzantium», Tesserae. Festschrift für Joseph Engemann, Münster («Jahrbuch für Antike und Christentum Ergänzungsband», 18), 1991, p. 74-92.

45. Philippe Buc, «Conversion of Objects: Suger of Saint Denis and Meinwerk of Paderborn», Viator, 28, 1997, p. 99-143. 
tandis que d'autres dorment dans une bibliothèque. Ils sont tous potentiellement des objets rituels, mais seuls certains le deviennent effectivement, et parfois seulement pour un temps. Il existe enfin des objets rituels qui cessent de fonctionner comme tels lorsqu'ils sont enfermés dans une vitrine de musée, réutilisés dans un autre contexte religieux ou lorsqu'ils sont sécularisés. Introduire la notion d'usage effectif dans la définition de l'objet rituel permet de limiter quelque peu la catégorie et de la rendre plus pertinente. Rappeler les changements de statut de certains objets rituels ajoute aussi une dynamique historique à l'histoire de la culture matérielle religieuse.

beatrice.caseau@paris-sorbonne.fr 